\title{
Molecular Cloning and Characterization of P4 Nuclease from Leishmania infantum
}

\author{
Safar Farajnia, ${ }^{1,2,3,4}$ Leila Rahbarnia, ${ }^{5}$ Bahram Maleki zanjani, ${ }^{1}$ \\ Mohammad Hossein Alimohammadian, ${ }^{6}$ Shahin Abdoli Oskoee, ${ }^{7}$ \\ Abbas Beh-pajooh, ${ }^{3}$ Nazli Saeedi, ${ }^{2}$ and Soheila Montazer Saheb ${ }^{4}$ \\ ${ }^{1}$ Biotechnology Research Center, Tabriz University of Medical Sciences, Tabriz 51656-65811, Iran \\ ${ }^{2}$ Drug Applied Research Center, Tabriz University of Medical Sciences, Tabriz 51656-65811, Iran \\ ${ }^{3}$ Infectious and Tropical Disease Research Center, Tabriz University of Medical Sciences, Tabriz 51656-65811, Iran \\ ${ }^{4}$ Tuberculosis and Lung Disease Research Center, Tabriz University of Medical Sciences, Tabriz 51656-65811, Iran \\ ${ }^{5}$ Deparetment of Agriculture, Zanjan University, Zanjan, Iran \\ ${ }^{6}$ Immunology Department, Pasteur Institute of Iran, Tehran, Iran \\ ${ }^{7}$ Faculty of Medicine, Tabriz University of Medical Sciences, Tabriz 51656-65811, Iran
}

Correspondence should be addressed to Safar Farajnia, farajnia@gmail.com and Leila Rahbarnia, le.rahbarnia@gmail.com

Received 3 January 2011; Revised 31 March 2011; Accepted 25 April 2011

Academic Editor: Ariel M. Silber

Copyright ( $) 2011$ Safar Farajnia et al. This is an open access article distributed under the Creative Commons Attribution License, which permits unrestricted use, distribution, and reproduction in any medium, provided the original work is properly cited.

\begin{abstract}
Parasite of the genus Leishmania is reliant on the salvage pathway for recycling of ribonucleotides. A class I nuclease enzyme also known as P4 nuclease is involved in salvage of purines in cutaneous Leishmania species but the relevant enzymes have not been characterized in Leishmania infantum (L. infantum). The aim of this study was to clone and characterize the gene encoding class I nuclease in L. infantum. DNA extracted from L. infantum was used for amplification of P4 nuclease gene (Li-P4) by PCR. The product was cloned, sequenced, and expressed in E. coli for further characterization. Analysis of the sequence of Li-P4 revealed that the gene consists of an ORF of 951 bp. Sequence similarity analysis indicated that Li-P4 has a high homology to relevant enzymes of other kintoplastids with the highest homology $(88 \%)$ to p1/s1 class I nuclease from L. donovani. Western blotting of antirecombinant Li-P4 with promastigote and amastigote stages of L. infantum showed that this nuclease is present in both stages of parasite with higher expression in amastigote stage. The highly conserved nature of this essential enzyme in Leishmania parasites suggests it as a promising drug target for leishmaniasis.
\end{abstract}

\section{Introduction}

Protozoan parasites of the genus Leishmania cause a spectrum of clinical disease, including cutaneous, mucocutaneous, and visceral leishmaniasis (VL). Approximately 12 million people are infected with this parasite worldwide with 1.5-2 million new cases occurring each year [1]. Leishmania parasites are dimorphic organisms which exist as promastigotes in extracellular stage and in the sandfly midgut, and as amastigote that lives intracellularly in the phagolysosomes of macrophages in the mammalian host cells [2, 3]. Unfortunately, currently available treatment regimens are nonselective drugs with significant toxicity and limited efficacy $[4,5]$. On the other hand, efforts aimed at the development of vaccines have only achieved low levels of protection in trials in human subjects $[6,7]$. Thus, there is an urgent need to identify novel molecular targets that can be exploited for drug development, vaccine design, or both. Purine salvage pathway seems as an attractive target for drug development against Leishmania. Trypanosomatid protozoa such as Leishmania are purine auxotrophs and are totally dependent upon their hosts to provide purine nucleotides for their survival, growth, and multiplication [8, 9]. It has shown that Leishmania promastigotes possess a unique class I nuclease, $[10,11]$ that involves the salvage of preformed purines through the hydrolysis of either $3^{\prime}$-nucleotides or nucleic acids [12-16]. An intracellular amastigote-specific protein, P-4, with class I nuclease activity has also been 
identified in L. pifanoi by Kar et al. [17] and by our group in L. major [18]. However, there was no data about the presence and characteristics of this nuclease in L. infantum, the causative agent of infantile human leishmaniasis. The aim of this study was to clone and characterize the P4 nuclease from Leishmania infantum.

\section{Materials and Methods}

2.1. Parasite and DNA Extraction. In this study, Iranian strain of Leishmania infantum was used. Promastigotes were cultured at $26^{\circ} \mathrm{C}$ in RPMI 1640 medium with glutamine (Gibco BRL) supplemented with 10\% heat-inactivated fetal calf serum (Sigma-Aldrich). Organisms were harvested in logarithmic phase and washed with phosphate buffer saline (PBS, pH 7.2). Parasites were disrupted in lysis buffer ( $50 \mathrm{mM} \mathrm{NaCl}, 50 \mathrm{mM}$ EDTA, 1\% SDS, $50 \mathrm{mM}$ Tris- $\mathrm{HCl}, \mathrm{pH}$ $8.0)$ and incubated overnight with proteinase $\mathrm{K}(100 \mathrm{mg} / \mathrm{mL}$, Sigma-Aldrich) at $37^{\circ} \mathrm{C}$. DNA was then purified by phenolchloroform extraction and ethanol precipitation.

2.2. PCR Amplification. A pair of primers was designed based on P4 gene sequence previously reported for cutaneous leishmaniasis (CL) strains: forward, $5^{\prime}$ CATATGTGGGGCTGCGTGGGTCACAT- $3^{\prime}$ and reverse, $5^{\prime}$ TACTCGAGCACCTCGCTTCGGACG-3' [19]. Each PCR reaction contained $200 \mathrm{ng}$ DNA, $10 \mathrm{p}$ mol each of forward and reverse primers, $1.5 \mathrm{mM} \mathrm{Mgcl} 2,200 \mu \mathrm{M}$ dNTPs, $1 \times$ PCR buffer, 2 unit of Pfu DNA polymerase (Fermentas) and up to $25 \mu \mathrm{L}$ d H2O. PCR amplification was carried out in $94^{\circ} \mathrm{C}$ for $4 \mathrm{~min}$, followed by 30 cycles of denaturation at $94^{\circ} \mathrm{C}$ for $1 \mathrm{~min}$, annealing at $60^{\circ} \mathrm{C}$ for $60 \mathrm{sec}$, and extension at $72^{\circ} \mathrm{C}$ for $60 \mathrm{sec}$ with a final extension cycle at $72^{\circ} \mathrm{C}$ for $20 \mathrm{~min}$. PCR products were electrophoresed on $1.5 \%$ agarose gel and stained by ethidium bromide. The DNA bands were visualized under an ultraviolet light (UV transilluminator) and documented.

2.3. Gene Cloning. The PCR product was purified by PCR product purification kit (Roche) and ligated into the pGEM-T easy vector (Promega). The ligation reaction was transformed into $\mathrm{DH} 5 \alpha$ (Promega) competent cells and plated on Luria-Bertani (LB) agar, containing ampicillin ( $50 \mathrm{mg} / \mathrm{mL})$, 5-bromo-4 chloro-3-indolyl- $\beta$-D-galactoside (X-gal: $20 \mathrm{mM}$ ), and Isopropyl thio- $\beta$-D-galactoside (IPTG: $200 \mathrm{mg} / \mathrm{mL}$ ). The white colonies containing recombinant plasmid were selected [20] for plasmid extraction and PCR screening [21]. Then cloning was verified by restriction digestion and sequencing.

2.4. Expression and Purification. The pGEM vector containing Li-P4 gene was digested with Nde I and Xho I and the insert was purified, subcloned into the Nde I - Xho I digested pET28a (Novagen) expression vector and transformed into the E. coli BL21 (Novagen). The bacteria containing pET28aLi-P4 was cultured in LB broth medium and grown until $\mathrm{OD}=0.5$. Expression of recombinant $\mathrm{P} 4$ was induced by addition of $25 \mu \mathrm{L}$ of $1 \mathrm{mM}$ IPTG then incubated for further $4 \mathrm{~h}$ at $37^{\circ} \mathrm{C}$ and analyzed by SDS-PAGE [22]. For

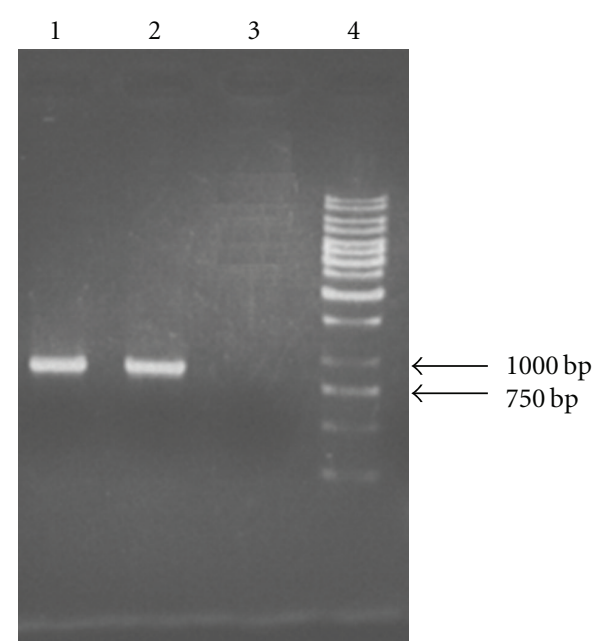

FIgURe 1: Amplification of P4 nuclease gene of Leishmania infantum. Lane 1 and Lane2: 951 bp Li-P4 gene PCR product, Lane 3: no DNA, and Lane 4: $1 \mathrm{~Kb}$ DNA Ladder.

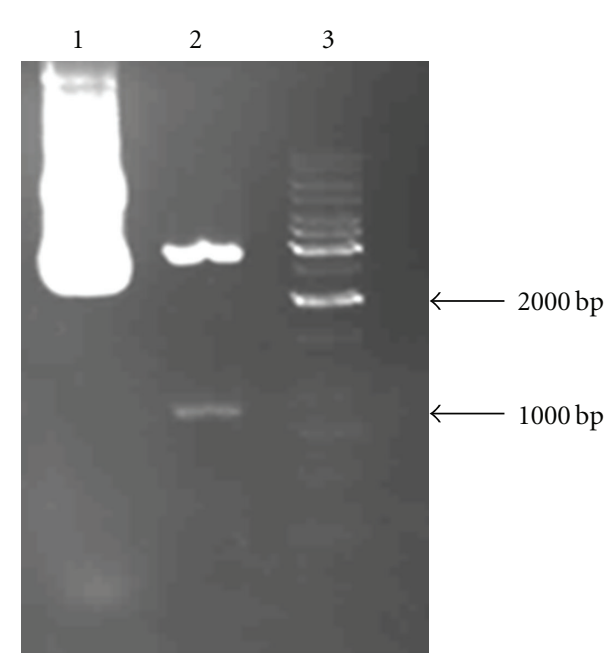

Figure 2: Cloning of Li-P4 nuclease gene in pGEM-t vector. Lane 1: undigested recombinant plasmid, Lane 2: Eco RI-digested recombinant plasmid, Lane 3: $1 \mathrm{~kb}$ DNA ladder.

purification of recombinant Li-P4, E. coli BL21 containing expression vector was cultured in 1 liter LB broth medium, and following induction with IPTG, the pellet was collected by centrifugation. The bacterial sediment was disrupted in $5 \mathrm{~mL}$ lysis buffer $(\mathrm{PH}=8,50 \mathrm{mM} \mathrm{NaH} 2 \mathrm{PO} 4,300 \mathrm{mM}$ $\mathrm{NaCl}$ ) by sonication, centrifuged for separation of soluble and insoluble fraction and analyzed by SDS-PAGE. Since the recombinant protein was expressed as 6 His-tag fusion, the Ni-NTA column (Qiagen) was used for purification. Briefly, Li-P4 inclusion body was dissolved in lysis buffer containing $8 \mathrm{M}$ urea and passed through Ni-NTA column that previously equilibrated with related buffer. The column was washed and recombinant protein was released and collected using $250 \mathrm{mM}$ Imidazol. The purified protein then was dialysed against PBS and used for further experiments. 


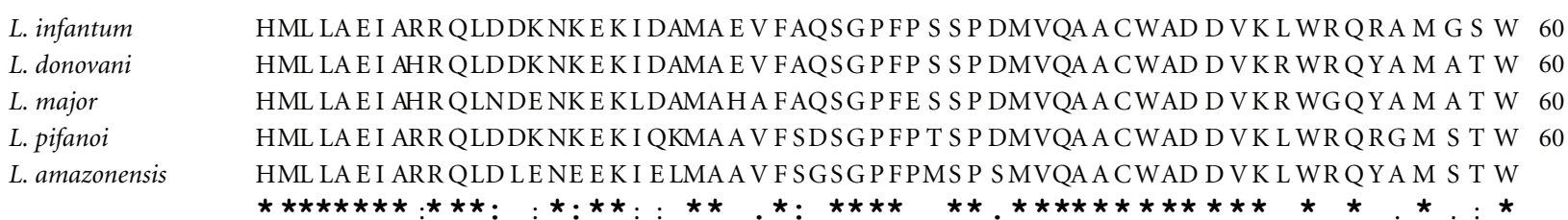

L. infantum

L. donovani

L. major

L. pifanoi

L. amazonensis

L. infantum

L. donovani

L. major

L. pifanoi

L. amazonensis

L. infantum

L. donovani

L. major

L. pifanoi

L. amazonensis

L. infantum
L. donovani
L. major
L. pifanoi
L. amazonensis

HML LA E I ARR QLDDKNK E K I DAMA E V FAQS G P FP S S P DMVQA A CWAD D V K L WR QRA M G S W 60 HML LA E I AHR QLDDK NK E K I DAMA E V FAQS G P FP S S P DMVQA A CWAD D V K R WR Q A M A T W 60 HML LA E I AHR QLNDE NK E KLDAMAHA FAQS G P F S S P DMVQA A CWAD D V K W WQY A M T W 60 HML LA E I ARR QLD LE NE E K I E LMA A V F S GS G P FPMS P S MVQA A CWAD D V K L WR QY A M S T W H YFDA PYNPEN IN I T DA I ATVNA VT V SRNMI SAL KNT KA P LYML N FAW AN L VH I F G D L H Q 120 H FFAA PYNPEN IN I T DA I DTVNAVT V S LDM I S L KNT K A P LYML N FAW AN L VH I FG D L H Q 120 H FFDK PYNP E D IN I T DP VATVNA VT V SRNMVTSLRRT NA P LY L L N FAW VN L V H I L D L H Q 120 H YYDKVYNPEN IN I T DP VNTVNA L T A SRNMVTS L KKS K A P LY L L N F AW VN L V H I F G D L H Q 120 H FYAMPYNPGN IN I T DP VNTVNA VT V CLDMVT S L KN KA P LY L L N F AW VN L V H I F G D L H Q 120

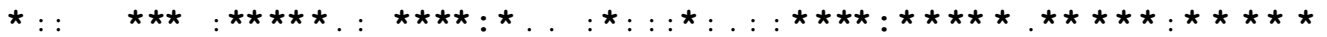

P LHT I SRYS S EYP HGDKGGQPLHT I VRGT S L SHGDK E A I CT G T P PR YQR P L S Y TD L F A L 180 P LHT I SRYS S EYP HGDKGGNL IQVMVGRKS L RLHA LWDN I CT GA P PR YQR P L S Y TD L F A L 180 P LHTT SRYS S EP HGDKGGNEVEVQVGKRKVNLHAVWDN I CS GT P PR Y KR P L S Y T L F A L 180 P LHT I SRYTT AYP HGDQGGNAVS VRAGRRKVKLHA LWDN I CT GA P PR YQR P L S TD L F A L 180 P LHT I SRYT T AYP HGDQGGNA I S VR VGGKKVKLHA LWDN I CS A T P PR YQR P L S HTD L F A L 180

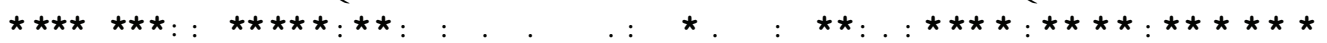

A ATADRL LET Y I F PE ALR TLVDVMA I HE E SHMFAVNT S Y PGVT P G A T L S E Y L A R K R V A 240 A ATADRL LET Y I F PE ALR TLVDVMA I HE E SHMFAVNT S Y PGVT P G A T L S E Y L A RC K R V A 240 A ATADRL LET YTF PE ALR TLVDVVA I HEE SHMFAVNT S Y PGVT P GAT L SDA Y L A R K R V A 240 S ATADRLVET YTF SE ELRTLVSVKA I HEE - YMFAVNT S Y PGVT P GAT L SDA Y L DKC K R V A 239 S ATADGLVET YTF SE AL E TLVDVMA I HEE S YMFAVNT S Y PGVT P GGT L SRA Y L D KC K R V A 240

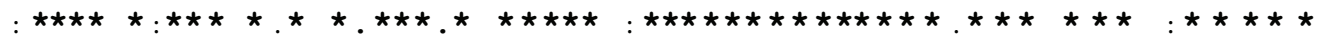

E ARL T LGGYR LG 252 E ARL T LGGYR LG 252 E ARL T LGGYR LG 252 E ARLT LGGYR LG 251 E ARL T LGGYR LG 252

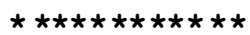

Figure 3: Alignment of deduced amino acid sequence of $L$. infantum amastigote P4 with class I nuclease from $L$. donovoni (GeneBank Accession no. ABE69185.1), L. major (Accession no. XP_001684745.1), L. amazonensis (Accession no. AAO65599.1), and L. pifanoi (Accession no. AAD48894.2). 5 conserved domains along with several semiconsereved domains between this nuclease are indicated by continuous stars.

Results of purification were controlled by \%10 SDS-PAGE gels.

2.5. Production of Rabbit Antiserum against Recombinant LiP4. An adult rabbit (New Zealand White) was immunized subcutaneously with $200 \mathrm{mg}$ of purified rL-iP4 emulsified in an equal volume of complete Freund's adjuvant (CFA) (Sigma-Aldrich), followed by a subcutaneous boosting 2 weeks later with $100 \mu \mathrm{g}$ of recombinant P4 protein in incomplete Freund's adjuvant (IFA) (Sigma-Aldrich). Two weeks later, the rabbit was boosted intravenously with $50 \mu \mathrm{g}$ of rLiP4, and blood was collected 1 week later.

2.6. Western Blotting. For determination of P4 nuclease, lysates of promastigotes and amastigotes of L. infantum were incubated with rabbit antiserum raised against rLi-P4 followed by incubation in 1/5000 dilution of HRP conjugated goat antirabbit IgG (Sigma-Aldrich). After washing to remove unbound antibodies, membranes were incubated in 1/5000 dilution of HRP conjugated goat antirabbit IgG
(Sigma) and processed for ECL. The protocol for detection of Leishmania elongation factor-1a (as an internal control) in lysates of promastigotes and amastigotes using mouse monoclonal anti-EF-1a and HRP-conjugated antimouse IgG was as described previously [23].

\section{Results and discussion}

3.1. Gene Cloning. After culture, the Leishmania infantum was subjected to DNA extraction and Li-P4 gene amplification. PCR amplification of P4 nuclease gene from L. infantum results in a PCR product of $951 \mathrm{bp}$ that was in expected size (Figure 1). The PCR product was cloned in the pGEM-T easy vector using T-A cloning method and confirmed by restriction digestion (Figure 2 ).

3.2. Sequencing and Multiple Alignments. Li-p4 was amplified from L. infantum amastigote using forward and reverse primers based on the sequence previously reported for cutaneous leishmaniasis (CL) strains. The PCR product was 


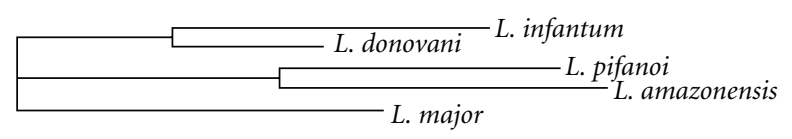

Figure 4: Phylogenetic tree of P1/S1 nuclease gene sequences among species of Leishmania. This tree shows the relatedness between P4 nuclease from $L$. infantum and P1/S1 nuclease $L$. donovani.

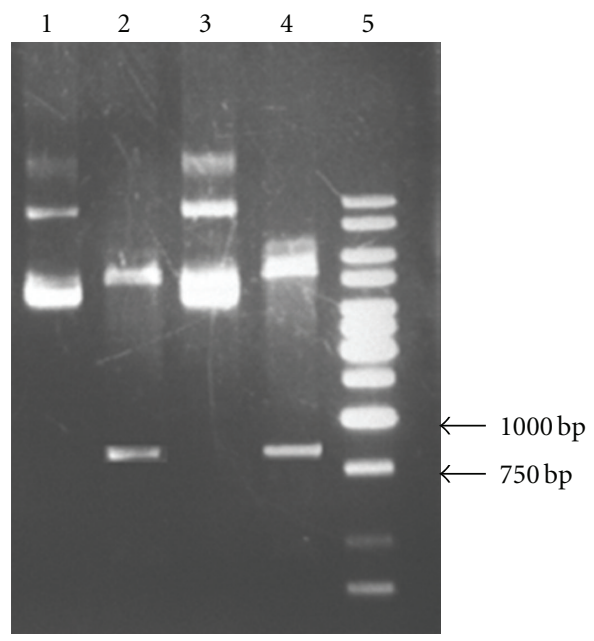

FIgURE 5: Cloning Li-P4 nuclease gene in pET 28a vector. Lane 1 and 3: recombinant plasmid, undigest, Lane 2 and 4: Nde I - Xho I digested recombinant plasmid, and Lane 5: $1 \mathrm{~Kb}$ DNA Ladder.

cloned into the pGEM-T easy vector and sequenced. The nucleotide sequence was submitted to the GeneBank/NCBI Data Base under accession number ABY27514.1. Result of sequencing revealed that the gene consists of an ORF of 951 base pairs with a predicted molecular mass of $33 \mathrm{kDa}$. Structural analysis using Signal P software showed a signal sequence consisting of the first 30 amino acids and two putative $\mathrm{N}$-linked glycosylation sites (at amino acid residues 108 and 251). A search of the SWISSPRO database with the predicted amino acid sequence revealed a significant similarity to a number of proteins belonging to the Class I nuclease family as shown by the alignment using the ClustalW program (Figure 3) [24]. The protein with highest similarity to $\mathrm{P} 4$ nuclease was $\mathrm{P} 1 / \mathrm{S} 1$ secretory nuclease of $L$. donovani (GenBank accession no. ABE69185.1) [23] (Figure 4). Other proteins with significant similarity were: $\mathrm{p} 1 / \mathrm{s} 1$ nuclease of L. major (accession no. XP_001684745.1) [18], singlestrand-specific nuclease from L. pifanoi (accession no. AAD48894.2), and P4 nuclease of L. amazonensis (accession no. AAO65599.1); identities and positivities between Li-P4 nuclease and each of these proteins are shown in Table 1. Analysis of the sequences for conserved domains using the NCBI protein blast tool showed that in spite of some heterogeneity in primary structure, all of these proteins contained a conserved nuclease domain specific to class I nuclease family.

3.3. Expression and Purification of Recombinant P4 Nuclease. For expression of recombinant Li-P4, the PCR product was

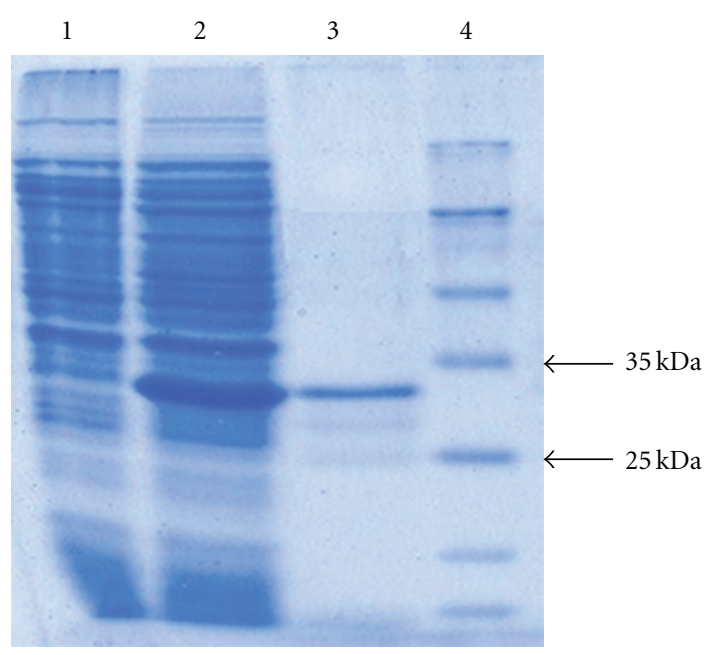

FIGURE 6: SDS-PAGE analysis of recombinant Li-P4 produced in BL21, Lane 1: bacterial lysate before induction, Lane 2: bacterial lysate after induction with IPTG, Lane 3: purified recombinant LiP4, and Lane 4: molecular weight marker.

TABLE 1: Similarity of predicted amino acid sequence of Li-P4 nuclease with different promastigote-specific P1/S1 nuclease of various Leishmania species.

\begin{tabular}{lcc}
\hline Leishmania species & Identity \%* & Positivity \%** \\
\hline P4 nuclease (Leishmania infantum) & 100 & 100 \\
$\begin{array}{l}\text { P1/S1 secretary nuclease (Leishmania } \\
\text { donovani) }\end{array}$ & 89 & 92 \\
$\begin{array}{l}\text { p1/s1 nuclease (Leishmania major) } \\
\text { single strand-specific nuclease }\end{array}$ & 81 & 89 \\
$\begin{array}{l}\text { (Leishmania pifanoi) } \\
\text { P4 nuclease (Leishmania } \\
\text { amazonensis) }\end{array}$ & 77 & 85 \\
\hline
\end{tabular}

${ }^{*}$ Identity is referred to the extent to which two sequences are invariant.

** Positivity is referred to the extent to which two sequences possess amino acids with the same physicochemical properties.

subcloned in the NdeI-XhoI site of pET28a (Novagen) in frame with C-terminal 6His-tag and transformed into the E. coli Bl21 (Figure 5). Induction of recombinant protein expression by IPTG resulted in a high level of expression. The molecular mass was found to be $33 \mathrm{kDa}$, which was slightly higher than the native mature protein due to the addition of a Hexa-His-tag sequence. Purification of recombinant protein was performed by affinity chromatography on Ni-NTA resin, and fractions were analyzed by SDS-PAGE (Figure 6).

\subsection{Differential Expression of P4 Protein in the Amastigotes.} To examine further developmental regulation of expression of P4 nuclease in L. infantum, polyclonal rabbit antisera raised against recombinant $\mathrm{Li}-\mathrm{P} 4$ were used to probe western blots containing lysates of promastigotes and amastigotes of L. infantum. As shown in Figure 7(a), a strongly reactive band of the expected mass $(33 \mathrm{kDa})$ was observed in lysates of amastigotes (lane 3) but appeared fairly in lysates of procyclic or metacyclic promastigotes (lane 1 and 2, resp.). 


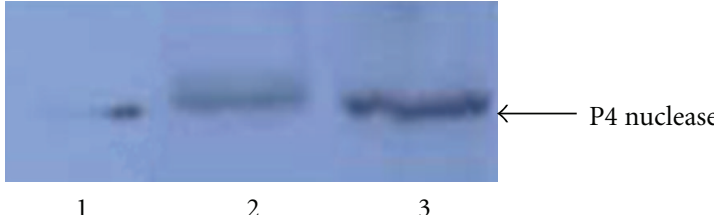

(a)

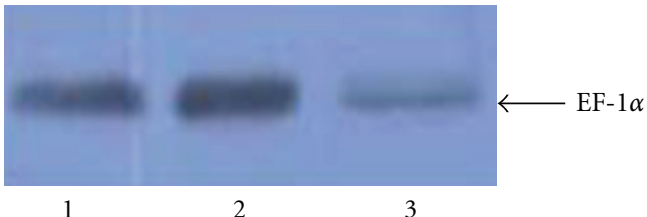

(b)

Figure 7: Analysis of P4 gene expression in different life cycle stages of L. infantum. (a) Promastigote and amastigote cell lysates were analyzed by immunoblotting using rabbit anti-recombinant Li-P4. Lane 1: Log phase promastigotes. Lane 2: Stationary phase promastigotes. Lane 3: Amastigotes. (b) The same blot as in a was probed with rabbit anti Elongation Factor 1-alfa antibody. The results indicate higher expression of $\mathrm{P} 4$ nuclease in amastigote stage of parasite.

To ensure that equivalent amounts of promastigote and amastigote proteins were loaded and transferred for detection, a similar blot was incubated with monoclonal antibody against EF- $1 \alpha$ (Upstate Biotechnology, USA), a protein that is expressed in all life cycle stages (Figure 7(b)). As it is shown in Figure 7(b), the abundance of EF- $1 \alpha$ in promastigote samples (lane 1 and 2) was similar to that one in amastigote lysates (lane 3 ).

$\mathrm{P}-4$ is a single-stranded specific class I nuclease that was identified initially in L. amazonensis [16] and then characterized in L. pifanoi by Kar et al. [17]. Recently, we isolated a class I nuclease gene from amastigotes of $L$. major [18]. In the present study we characterized this nuclease in L. infantum. Similarity analysis revealed that the sequence of corresponding protein from $L$. infantum has high sequence homology to the $\mathrm{P} 4$ nuclease of $L$. donovani, $L$. major, L. Pifanoi, and $3^{\prime}$-nucleotidase/nuclease enzymes previously described in different trypanosomatids [11]. The alignment results indicated the presence of 5 main conserved domains between these nucleases from different species.

The class I nuclease from promastigote stage of some Leishmania species have been extensively studied $[8,13,19$, $25,26]$. It has shown that expression of this nuclease upregulated significantly in response to purine starvation $[13,17]$ which is consistent with the opinion that class I nuclease enzyme plays a vital role in providing purines for growth and development of Leishmania parasites. Since there is no a homologue enzyme with similar properties in mammalian tissues, this enzyme can serve as a chemotherapeutic target for selective targeting of Leishmania during infection [11]. Considering that Leishmania is present as amastigotes in mammalian tissues and this stage is responsible for disease manifestations, the higher expression of the class I nuclease in amastigote stage further supports the use of this enzyme as a target.

\section{Conclusion}

In conclusion, the results of the present study revealed that Li-P4 nuclease belongs to the class I nuclease group of enzymes that are highly expressed in amastigote stage of $L$. infantum and could be exploited as target for chemotherapy.

\section{References}

[1] P. Desjeux, "Leishmaniasis: current situation and new perspectives," Comparative Immunology, Microbiology and Infectious Diseases, vol. 27, no. 5, pp. 305-318, 2004.

[2] A. Campos-Neto, R. Porrozzi, K. Greeson et al., "Protection against cutaneous leishmaniasis induced by recombinant antigens in murine and nonhuman primate models of the human disease," Infection and Immunity, vol. 69, no. 6, pp. 4103-4108, 2001.

[3] K. Campbell, H. Diao, J. Ji, and L. Soong, "DNA immunization with the gene encoding $\mathrm{p} 4$ nuclease of Leishmania amazonensis protects mice against cutaneous leishmaniasis," Infection and Immunity, vol. 71, no. 11, pp. 6270-6278, 2003.

[4] J. E. Jackson, J. D. Tally, W. Y. Ellis et al., "Quantitative in vitro drug potency and drug susceptibility evaluation of Leishmania ssp. from patients unresponsive to pentavalent antimony therapy," American Journal of Tropical Medicine and Hygiene, vol. 43, no. 5, pp. 464-480, 1990.

[5] M. Grogl, T. N. Thomason, and E. D. Franke, "Drug resistance in leishmaniasis: its implication in systemic chemotherapy of cutaneous and mucocutaneous disease," American Journal of Tropical Medicine and Hygiene, vol. 47, no. 1, pp. 117-126, 1992.

[6] I. Sharifi, A. R. Fekri, M. R. Aflatonian et al., "Randomised vaccine trial of single dose of killed Leishmania major plus BCG against anthroponotic cutaneous leishmaniasis in Bam, Iran," Lancet, vol. 351, no. 9115, pp. 1540-1543, 1998.

[7] E. A. G. Khalil, A. M. El Hassan, E. E. Zijlstra et al., "Autoclaved Leishmania major vaccine for prevention of visceral leishmaniasis: a randomised, double-blind, BCG-controlled trial in Sudan," Lancet, vol. 356, no. 9241, pp. 1565-1569, 2000.

[8] H. F. Hassan and G. H. Coombs, "A comparative study of the purine- and pyrimidine-metabolising enzymes of a range of trypanosomatids," Comparative Biochemistry and Physiology_Part B, vol. 84, no. 2, pp. 219-223, 1986.

[9] C. Bryant and C. A. Behm, Biochemical Adaptation in Parasites, Chapman and Hall, New York, NY, USA, 1989.

[10] J. B. Sacci, T. A. Campbell, and M. Gottlieb, "Leishmania donovani: regulated changes in the level of expression of the surface 3'-nucleotidase/nuclease," Experimental Parasitology, vol. 71, no. 2, pp. 158-168, 1990.

[11] W. F. Sopwith, A. Debrabant, M. Yamage, D. M. Dwyer, and P. A. Bates, "Developmentally regulated expression of a cell surface class I nuclease in Leishmania mexicana," International Journal for Parasitology, vol. 32, no. 4, pp. 449-459, 2002. 
[12] D. J. Hammond and W. E. Gutteridge, "Purine and pyrimidine metabolism in the Trypanosomatidae," Molecular and Biochemical Parasitology, vol. 13, no. 3, pp. 243-261, 1984.

[13] M. Gottlieb, "Enzyme regulation in a trypanosomatid: effect of purine starvation on levels of 3'-nucleotidase activity," Science, vol. 227, no. 4682, pp. 72-74, 1985.

[14] M. Gottlieb and D. M. Dwyer, "Evidence for distinct 5'- and 3 '-nucleotidase activities in the surface membrane fraction of Leishmania donovani promastigotes," Molecular and Biochemical Parasitology, vol. 7, no. 4, pp. 303-317, 1983.

[15] D. M. Dwyer and M. Gottlieb, "Surface membrane localization of 3'- and 5'-nucleotidase activities in Leishmania donovani promastigotes," Molecular and Biochemical Parasitology, vol. 10, no. 2, pp. 139-150, 1984.

[16] L. Soong, S. M. Duboise, P. Kima, and D. McMahon-Pratt, "Leishmania pifanoi amastigote antigens protect mice against cutaneous leishmaniasis," Infection and Immunity, vol. 63, no. 9, pp. 3559-3566, 1995.

[17] S. Kar, L. Soong, M. Colmenares, K. Goldsmith-Pestana, and D. McMahon-Pratt, "The immunologically protective P-4 antigen of Leishmania amastigotes. A developmentally regulated single strand-specific nuclease associated with the endoplasmic reticulum," Journal of Biological Chemistry, vol. 275, no. 48, pp. 37789-37797, 2000.

[18] S. Farajnia, M. H. Alimohammadian, N. E. Reiner, M. Karimi, S. Ajdari, and F. Mahboudi, "Molecular characterization of a novel amastigote stage specific class I nuclease from Leishmania major," International Journal for Parasitology, vol. 34, no. 8, pp. 899-908, 2004.

[19] J. D. Thompson, D. G. Higgins, and T. J. Gibson, "CLUSTAL $\mathrm{W}$ : improving the sensitivity of progressive multiple sequence alignment through sequence weighting, position-specific gap penalties and weight matrix choice," Nucleic Acids Research, vol. 22, no. 22, pp. 4673-4680, 1994.

[20] A. L. Bothwell, G. D. Yancopulos, and F. W. Alt, Methods for Cloning and Analysis of Eukaryotic Genes, vol. 10, Jones and Bartlett, Boston, Mass, USA, 1990.

[21] I. Feliciello and G. Chinali, "A modified alkaline lysis method for the preparation of highly purified plasmid DNA from Escherichia coli," Analytical Biochemistry, vol. 212, no. 2, pp. 394-401, 1993.

[22] E. Salehi, S. Farajnia, K. Parivar et al., "Recombinant expression and purification of L2 domain of human epidermal growth factor receptor," African Journal of Biotechnology, vol. 9, no. 33, pp. 5292-5296, 2010.

[23] S. Farajnia, F. Mahboudi, S. Ajdari, N. E. Reiner, A. Kariminia, and M. H. Alimohammadian, "Mononuclear cells from patients recovered from cutaneous leishmaniasis respond to Leishmania major amastigote class I nuclease with a predominant Th1-like response," Clinical and Experimental Immunology, vol. 139, no. 3, pp. 498-505, 2005.

[24] T. A. Campbell, G. W. Zlotnick, T. A. Neubert, J. B. Sacci, and M. Gottlieb, "Purification and characterization of the 3'-nucleotidase/nuclease from promastigotes of Leishmania donovani," Molecular and Biochemical Parasitology, vol. 47, no. 1, pp. 109-117, 1991.

[25] G. O. Gbenle, F. R. Opperdoes, and J. Van Roy, "Localization of 3'-nucleotidase and calcium-dependent endoribonuclease in the plasma-membrane of Trypanosoma brucei," Acta Tropica, vol. 43, no. 4, pp. 295-305, 1986.

[26] M. Gottlieb, P. R. Gardiner, and D. M. Dwyer, “3'-nucleotidase activity in procyclic and bloodstream stages of Trypanosoma rhodesiense," Comparative Biochemistry and Physiology-Part $B$, vol. 83, no. 1, pp. 63-69, 1986. 

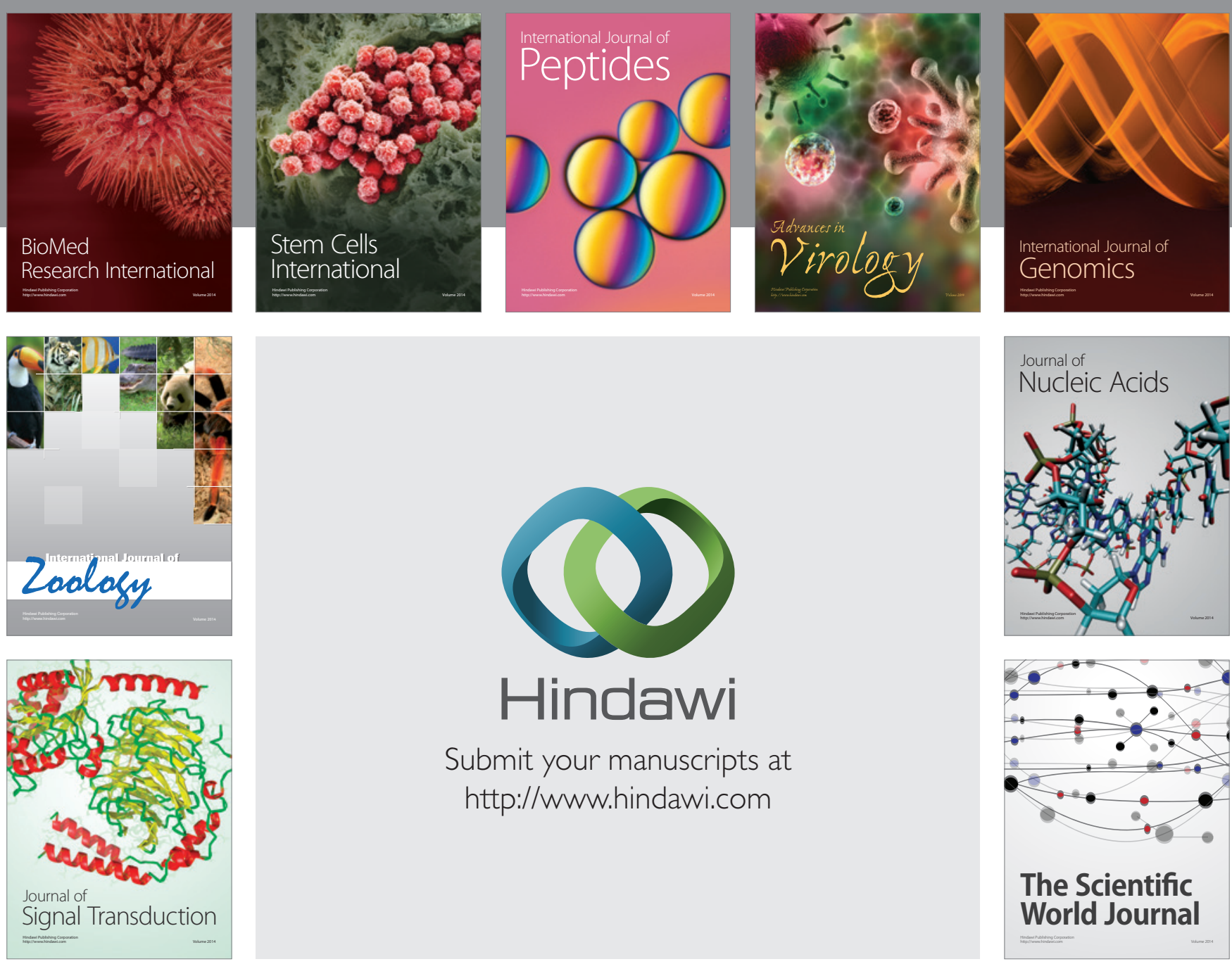

Submit your manuscripts at

http://www.hindawi.com
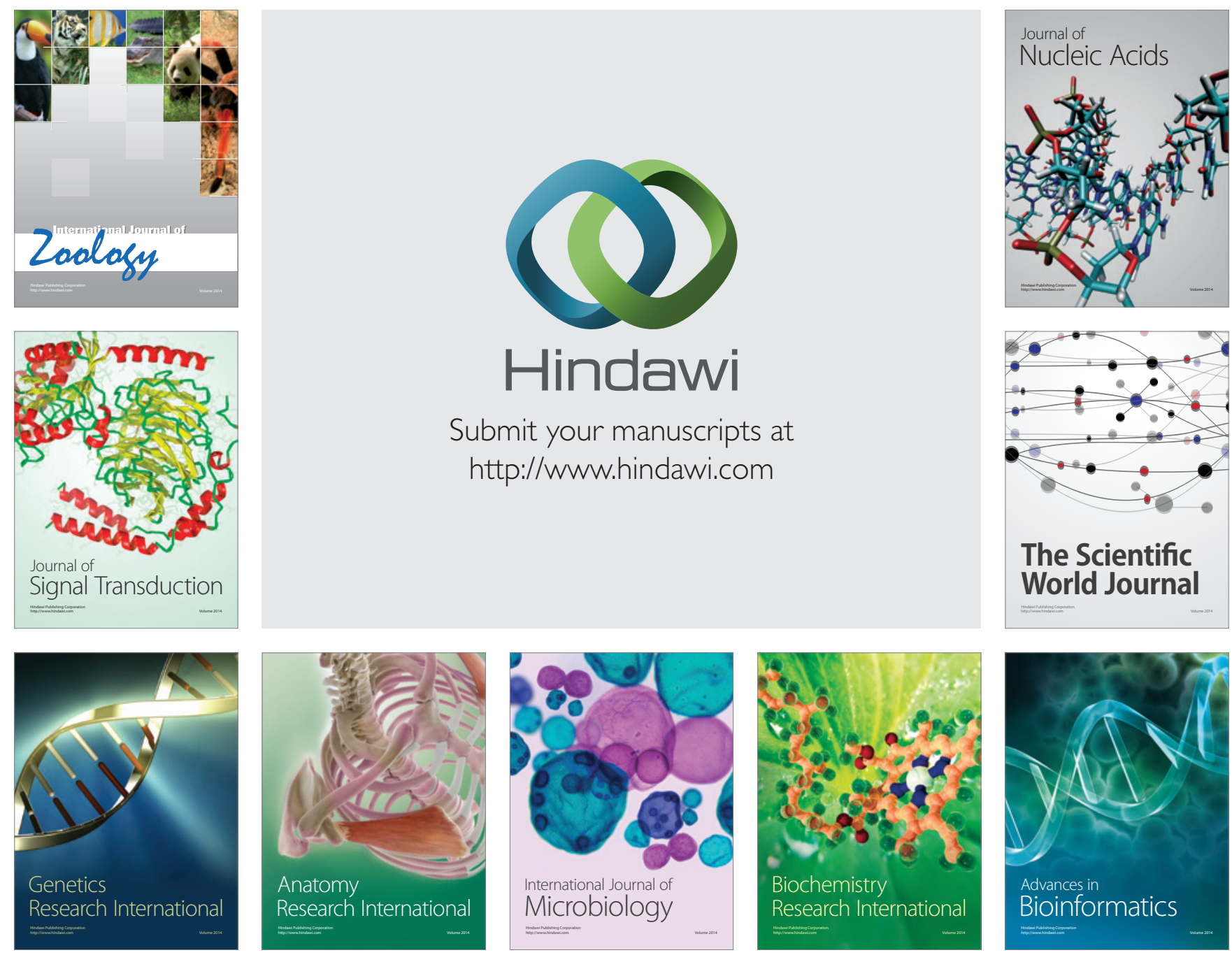

The Scientific World Journal
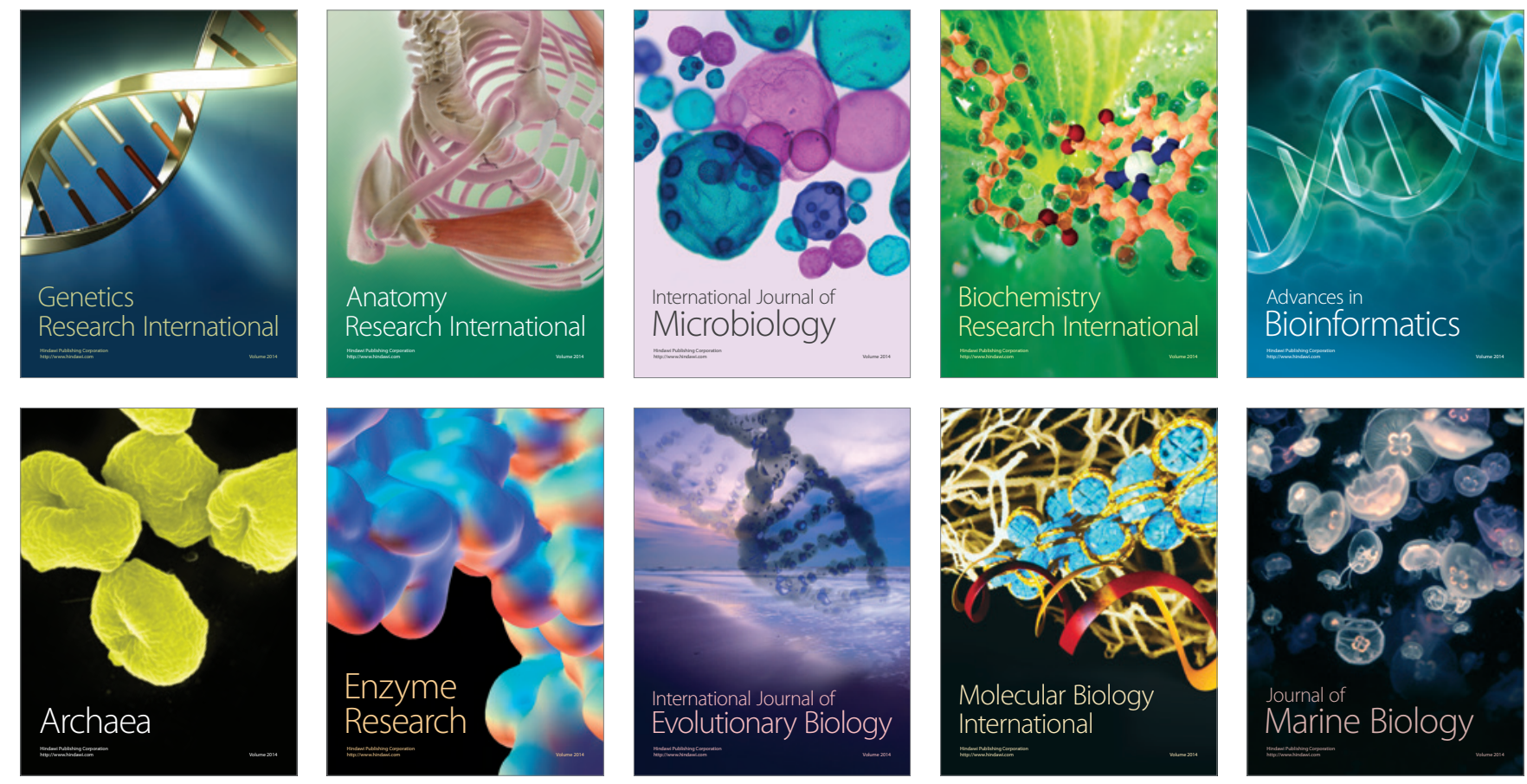